\title{
COLOR GRADIENTS IN EARLY-TYPE GALAXIES: DEPENDENCE ON ENVIRONMENT AND REDSHIFT
}

\author{
F. La Barbera, ${ }^{1}$ R. R. de Carvalho, ${ }^{2}$ R. R. Gal, ${ }^{3}$ G. Busarello, ${ }^{1}$ P. Merluzzi, ${ }^{1}$ M. Capaccioli, ${ }^{1}$ and S. G. Djorgovski ${ }^{4}$ \\ Received 2004 December 23; accepted 2005 April 29; published 2005 May 23
}

\begin{abstract}
Color gradients in early-type galaxies contain valuable clues about their formation and evolutionary histories and mechanisms. We examine color gradients in 1700 early-type galaxies in 159 galaxy clusters spanning a redshift range of $0.05-0.2$. We find that color gradients strongly depend on the environment where galaxies reside, with steeper color gradients in poor rather than rich clusters. No dependence of color gradients on galaxy luminosity is found in either rich or poor clusters. The difference in color gradients can be explained by a change in the internal metallicity and/or an age gradient in these galaxies. Our results support a reasonable picture whereby young early-type galaxies form in a dissipative collapse process and then undergo increased (either major or minor) merging activity in richer rather than in poor clusters.
\end{abstract}

Subject headings: galaxies: clusters: general — galaxies: evolution — galaxies: formation — galaxies: fundamental parameters

\section{INTRODUCTION}

Early-type galaxies (ETGs) have negative internal color gradients, with their stellar populations becoming bluer toward the galaxy outskirts. Different studies have shown that these gradients are mainly caused by a metallicity variation (e.g., Peletier et al. 1990, hereafter PDI90). As reviewed by PDI90, color gradients can be used as an effective discriminant among galaxy formation scenarios. In a monolithic collapse picture, ETGs form by the rapid collapse of overdense regions at high redshift. Galactic winds are expected to produce steeper metallicity gradients in more massive galaxies (Larson 1974; Carlberg 1984). On the other hand, merging is expected to produce some dilution of the gradients in galaxies (White 1980), deleting or producing an inverse correlation among stellar population gradients and luminosity, and making the gradients less steep in denser environments.

It is also unclear whether or not color gradients in ETGs depend on environment and/or galaxy luminosity. Tamura \& Ohta (2003, hereafter TaO03) found the mean color gradient of ETGs in a nearby cluster to be consistent with the value obtained for field galaxies by other authors (PDI90: Idiart et al. 2002, hereafter IdM02). On the other hand, Tamura \& Ohta (2000) and Tamura et al. (2000, hereafter TKA00) studied color gradients in both cluster and field galaxies at intermediate redshifts and found that the former seem to have less steep gradients. To date, no study of color gradients for galaxies in groups has been performed.

Kormendy \& Djorgovski (1989) examined the dependence of color gradients on luminosity and other galaxy properties. While no clear trend with luminosity was found, they noted that the largest gradients occur in intermediate luminosity galaxies and suggested that postformation mergers on average diminish the primordial gradients in the more luminous systems; this was also supported by their finding that galaxies with more anisotropic velocity distributions have weaker color gradients. TaO03 also showed that for cluster galaxies some evi-

\footnotetext{
${ }^{1}$ INAF, Osservatorio Astronomico di Capodimonte, Naples, Italy; labarber@na.astro.it.

2 INPE/MCT, Avenida dos Astronautas, 1758, São José dos Campos, SP 12227-010, Brazil.

${ }^{3}$ Department of Physics, University of California at Davis, 1 Shields Avenue, Davis, CA 95616.

${ }^{4}$ Department of Physics, Mathematics, and Astronomy, California Institute of Technology, MS 105-24, Pasadena, CA 91125.
}

dence exists for a bimodal behavior, with very bright $\left(L>L^{*}\right)$ ETGs having gradients that steepen with luminosity, and fainter galaxies showing the opposite trend. La Barbera et al. (2004) and de Propris et al. (2004) found that gradients in cluster ETGs do not change or can become more steep at fainter luminosities.

In this Letter, we study the dependence of $g-r$ color gradients in ETGs on galaxy luminosity and environment. Color gradients are estimated by deriving structural parameters, namely the effective radius, $r_{e}$, and the Sersic index, $n$, for a sample of 1700 ETGs in 159 clusters $^{5}$ with different richnesses in the redshift range $0.05 \lesssim z \lesssim 0.2$. We assume a $\Lambda \mathrm{CDM}$ cosmology with $\Omega_{m}=0.3, \Omega_{\Lambda}=0.7$, and $H_{0}=70 \mathrm{~km} \mathrm{~s}^{-1} \mathrm{Mpc}^{-1}$.

\section{DATA}

The data are taken from the Palomar Abell Cluster Survey (Gal et al. 2000) and were collected at the $1.5 \mathrm{~m}$ telescope at Palomar with a SITe $2048 \times 2048$, AR-coated CCD. The pixel scale at this detector is 0.368 pixel $^{-1}$, yielding a $12.56 \times$ 12 '.56 field of view. Data were taken in $g, r$, and $i$ Gunn-Thuan filters. We refer the reader to Gal et al. (2000) for more details on data reduction and photometric quality.

Galaxies used for this study were selected as follows (see F. La Barbera et al. 2005, in preparation, for details). We selected likely cluster members as those galaxies whose $g-r$ colors are within $\pm \alpha \sigma_{\mathrm{CM}}$ around the color-magnitude relation of each cluster, where $\sigma_{\mathrm{CM}}$ is the observed dispersion of the red sequence. Field contamination was estimated using 34 blank fields observed with the same configuration and exposure times as the clusters. In order to keep field contamination as small as possible without overly reducing the total number of ETGs, we use $\alpha=0.5$. From the 34 blank fields, we measure the mean number of field galaxies found in the same magnitude and color range used to select galaxies in each of the clusters. Choosing $\alpha=0.5$, the fraction of field galaxies was typically $\sim 10 \%$ for the entire cluster sample. We note that decreasing the value of $\alpha$ reduces dramatically the total number of ETGs without changing significantly the field contamination. For example, choosing $\alpha=0.25$ decreases the number of ETGs by $40 \%$, while the fraction of expected field galaxies decreases by only $1 \%$. On the other hand, increasing the value of $\alpha$ makes field contamination significantly higher. For example, choosing

\footnotetext{
${ }^{5}$ The list of clusters is available from the first author.
} 


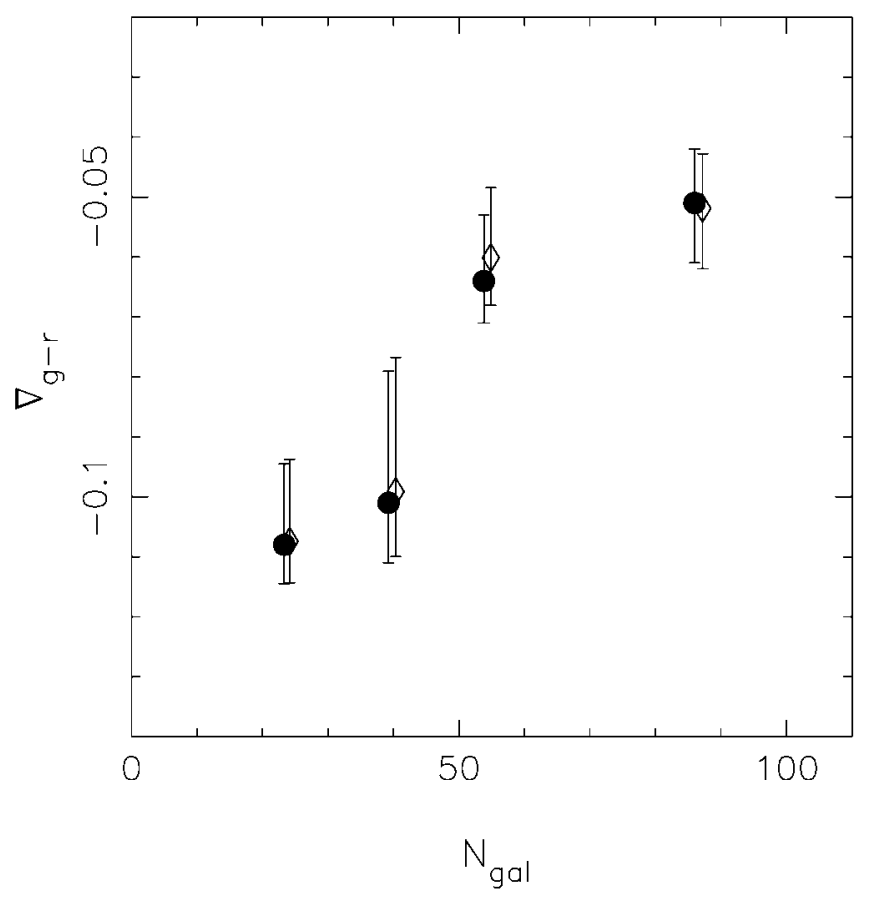

FIG. 1.-For clusters with $z<0.15$ and well-measured richnesses, we plot the mean value of the $g-r$ color gradients, $\nabla_{g-r}$ vs. the mean cluster richness, $N_{\text {gal }}$ (filled symbols). Each bin includes $N=140$ galaxies. Open circles are the mean values of $\nabla_{g-r}$ corrected for field contamination. Error bars denote $1 \sigma$ uncertainties. A small offset in richness has been added to separate the corrected and uncorrected data points.

$\alpha=0.75,1.0$, or 1.5 , the fraction of field galaxies increases to $16 \%, 18 \%$, or $20 \%$, respectively.

The value of $\sigma_{\mathrm{CM}}$ was obtained for each cluster by fixing the red sequence slope to the value given by Visvanathan \& Sandage (1977) at the corresponding redshift and by computing a $3 \sigma$ clipped standard deviation of residuals to the red sequence. We excluded galaxies with overlapping isophotes, which were defined using an isophotal level of $1.5 \sigma$ of the background standard deviation. These objects could be interacting galaxies at the cluster redshift or objects at different redshifts that overlap because of projection effects. This selection minimizes cases in which color gradients might be driven by interaction with nearby objects rather than by global cluster and/or galaxy properties. For each cluster, we also selected only galaxies brighter than a given magnitude limit. We simulated galaxy images as a function of redshift and calculated the necessary signal-to-noise ratio $(\mathrm{S} / \mathrm{N})$ to recover the $g-r$ color gradients with systematic errors less than 0.005 mag (La Barbera et al. 2002, hereafter LBM02). For each cluster, we set the magnitude limit such that this $\mathrm{S} / \mathrm{N}$ is achieved in the radial range used (see below). The typical value for the magnitude cut was $r=18.5$.

The above criteria yield a sample of 1950 galaxies for which structural parameters were derived in the $g$ and $r$ bands. From these galaxies, we selected the 1700 objects with Sersic index $n>2.2$, which are likely cluster ETGs (Blanton et al. 2003). For each cluster, we measure the richness from Digitized Palomar Observatory Sky Survey (DPOSS) galaxy catalogs (Gal et al. 2003). Only 108 of the clusters (containing 1135 ETGs) have well-calibrated DPOSS data. Additionally, a number of systematic effects could introduce a bias into our results at higher redshift. These effects include the enhanced detectability of rich clusters as opposed to poor ones, the faintness and smaller sizes of the galaxies in high- $z$ clusters, and sampling of a different portion of the rest-frame galaxy spectra in the $g$ and $r$ filters. Therefore, for the richness-dependent analyses we further restrict the data set to the 560 galaxies in 75 wellmeasured clusters with $z<0.15$.

\section{DERIVATION OF COLOR GRADIENTS}

Surface photometry was derived by fitting galaxy stamps with PSF-convolved Sersic models, as detailed in LBM02. For each stamp, a constant background value was fitted simultaneously with the model, achieving a typical accuracy of better than $0.1 \%$ in the background value. For each cluster and in each filter, the point-spread function (PSF) model was constructed by fitting star images with a sum of three Moffat functions, giving a mean value of $\sim 1$ for the reduced $\chi^{2}$ of the PSF fits in the whole sample. Deviations of the PSF from a circular shape were also taken into account by expanding stellar isophotes in a cos and sin series. Details will be given in a forthcoming paper (F. La Barbera et al. 2005, in preparation). The best-fitting structural parameters $(r$ and $n)$ in the $g$ and $r$ bands were used to derive the internal color profile for each galaxy, $g-r(r)$, where $r$ is the distance from the galaxy center. The color gradient, defined as $\nabla_{g-r}=\delta(g-r) / \delta(\log r)$, was estimated by the logarithmic slope of the color profile, performing a linear fit of $g-r(r)$ versus $\log r$. For comparison with other works (e.g., PDI90), the fits were performed in the range $r_{\min }<r<r_{\max }$, with $r_{\min }=0.1 r_{e}$ and $r_{\max }=r_{e}$, where $r_{e}$ is the effective radius in the $r$ band. The typical value of $r_{e}$ for the galaxies analyzed here varies from $\sim 1$ ". 9 at $z<0.15$ to $\sim 1$.'1 at higher redshifts. We also note that slightly varying (by $\sim 30 \%$ ) the values of $r_{\min }$ and/or $r_{\max }$ changes the $\nabla_{g-r}$ values by less than $0.01 \mathrm{mag}$, demonstrating the robustness of our results.

\section{RESULTS}

Galaxies were binned according to their parent cluster richnesses, each bin having the same number of galaxies. As noted in $\S 2$, we use only the $N=560$ galaxies in clusters at $z<$ 0.15 , which is the median redshift of the whole sample. Figure 1 shows the mean color gradient (filled symbols) as a function of the mean cluster richness $N_{\text {gal }}$. The figure clearly shows that $\nabla_{g-r}$ becomes less steep for galaxies in richer clusters. The difference in $\nabla_{g-r}$ between points with $N_{\text {gal }}<50$ and $N_{\text {gal }}>50$ is $-0.047 \pm 0.008$ and is significant at $\sim 5.9 \sigma$. In order to estimate the effect of field contamination on the $\nabla_{g-r}$ versus $N_{\text {gal }}$ relation, we used a Monte Carlo technique. For each cluster, we measure $N_{c}$ galaxies, which are likely to be ETGs. From the blank fields we then measure $N_{f}$ galaxies, which follow the same set of criteria for being in the cluster-these are the contaminants. To account for possible magnitude-dependent differences between galaxy populations, the cluster and field galaxies are divided into three magnitude bins, and in each bin $i$ we randomly select a total of $N_{c, i}-N_{f, i}$ distinct galaxies that are expected to be cluster members. This procedure was iterated 500 times for all of the clusters, recomputing at each iteration the mean color gradient in each richness range. Figure 1 plots the mean value of the corrected $\nabla_{g-r}$ values as open symbols. The corresponding error bars are obtained by adding in quadrature the standard deviation of the background-corrected $\nabla_{g-r}$ values to the errors on the uncorrected gradients. The difference between the corrected points with $N_{\text {gal }}<50$ and $N_{\text {gal }}>50$ is $-0.047 \pm 0.009$, which is significant at $\sim 5.5 \sigma$. We conclude that field contamination has no effect on our results. 


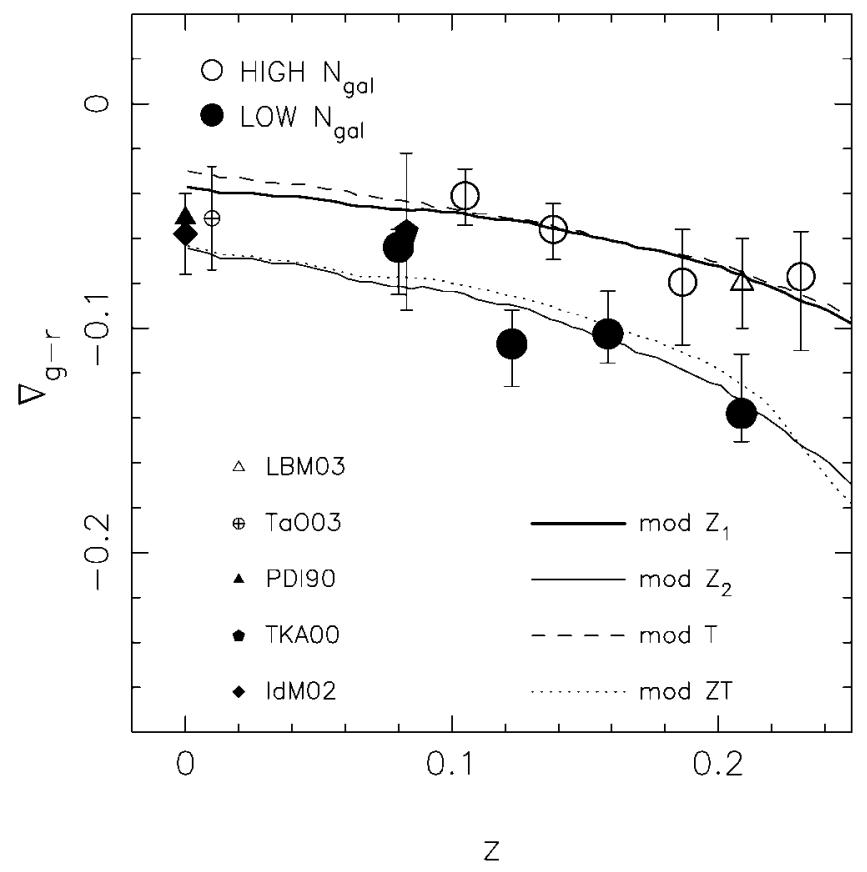

FIG. 2.-Mean $g-r$ color gradient, $\nabla_{g-r}$, is plotted vs. redshift for high- and low-richness clusters (open and filled circles, respectively). Error bars denote $1 \sigma$ uncertainties. Gradients from the literature for field and cluster ETGs are plotted as filled and open symbols, respectively (see references in the figure). Curves represent the stellar population models for the color gradients.

Figure 2 shows the mean value of $\nabla_{g-r}$ in different redshift bins. The whole sample was divided into low $\left(N_{\mathrm{gal}}<50\right)$ and high $\left(N_{\text {gal }}>50\right)$ richness clusters (hereafter LRCs and HRCs, respectively), with similar numbers of galaxies in both subsamples. The gradients for each subsample are given in Table 1. In Figure 2 we also show optical color gradients from previous works for field (open symbols) and cluster (filled symbols) ETGs at $z \lesssim 0.2$. The gradients were transformed to $\nabla_{g-r}$ values using the GISSEL03 spectral code (Bruzual \& Charlot 2003), as detailed in F. La Barbera et al. (2005, in preparation). We see that our data are in good agreement with previous measurements of color gradients. Figure 2 shows that color gradients slightly decrease with redshift, following two distinct trends depending on the cluster richness. To quantify these trends, we performed a linear fit of $\nabla_{g-r}$ versus $z$ for both LRCs and HRCs, using a least squares procedure with $\nabla_{g-r}$ as the dependent variable. The uncertainties on the fitting coefficients were obtained by shifting points in Figure 2 according to the corresponding error bars and repeating the fitting procedure. The slopes of the fitted lines are $-0.52 \pm 0.18$ for LRCs and $-0.32 \pm 0.2$ for HRCs. These values are negative at 2.9 and $1.6 \sigma$ significance levels, respectively. We note that the difference between $\nabla_{g-r}$ of LRCs and HRCs in Table 1 is $-0.043 \pm 0.008$, which is significant at $\sim 5 \sigma$ (see above).

To constrain the underlying age and metallicity gradients in the galaxies, we used four empirical stellar population models for $\nabla_{g-r}$ : two pure metallicity models $\left(Z_{1}\right.$ and $\left.Z_{2}\right)$, a pure age model $(T)$, and a mixed age + metallicity model (TZ). Each model was constructed by computing the difference in color between two simple stellar populations, describing the properties of stellar populations at the inner and outer galaxy radii, $r_{\min }$ and $r_{\max }$, used for the computation. Galaxy colors were obtained from the GISSEL03 code. The inner population was assumed to be old ( $T=12 \mathrm{Gyr}$ ) with solar metallicity, while the age and metallicity of the outer population were changed to reproduce the actual color gradients. In models $Z_{1}$ and $Z_{2}$
TABLE 1

\begin{tabular}{|c|c|}
\hline $\begin{array}{l}\text { MEan Color } \\
\text { GalaXIES IN } \\
\text { DifFERENT }\end{array}$ & $\begin{array}{l}\text { ADIENTS FOF } \\
\text { STERS WITH } \\
\text { HNESSES }\end{array}$ \\
\hline$z$ & $\nabla_{g-r}$ \\
\hline & \\
\hline 0.08 & $-0.064_{0.02}^{0.008}$ \\
\hline 0.12 . & $-0.106_{0.02}^{0.015}$ \\
\hline 0.16 & $-0.103_{0.013}^{0.02}$ \\
\hline $0.21 \ldots$ & $-0.137_{0.014}^{0.026}$ \\
\hline & \\
\hline 0.105 & $-0.04_{0.013}^{0.012}$ \\
\hline $0.14 \ldots \ldots \ldots \ldots$ & $-0.056_{0.013}^{0.012}$ \\
\hline $0.19 \ldots \ldots \ldots$ & $-0.08_{0.026}^{0.024}$ \\
\hline $0.23 \ldots \ldots \ldots$ & $-0.079_{0.027}^{0.026}$ \\
\hline
\end{tabular}

the outer populations are old, but their metallicities are changed in order to describe the color gradients in HRCs and LRCs, respectively. Model $T Z$ was created to describe the gradients in poorer clusters by adding an age gradient to the metallicity gradient of model $Z_{1}$, which reproduces the color gradient in richer clusters. In model $T$, the inner and outer stellar populations have the same metallicity, while the age gradient is chosen to reproduce the color gradient in rich clusters. Table 2 reports the relevant parameters of each model, together with the corresponding metallicity and age gradients, $\nabla_{Z}=$ $\log \left(Z_{o} / Z_{i}\right)$ and $\nabla_{T}=\log \left(T_{o} / T_{i}\right)$, respectively.

Figure 3 plots the mean value of $\nabla_{g-r}$ for galaxies at $z<$ 0.15 as a function of their absolute $r$ magnitude, which was obtained by using $k$-corrections from the GISSEL03 code. Three samples are shown in the plot, corresponding to galaxies in clusters with low, high, and all richnesses, respectively. We see that color gradients do not depend significantly on galaxy luminosity.

\section{DISCUSSION}

The dependence of color gradients on cluster richness can be explained by the fact that some physical mechanism, related to galaxy environment, affects the age and metallicity gradients in ETGs. A pure metallicity gradient can account for the observed color gradients, provided that $\nabla_{Z}$ flattens from about $-0.4\left(\operatorname{model} Z_{2}\right)$ in LRCs to $\sim-0.2\left(\operatorname{model} Z_{1}\right)$ in HRCs. These metallicity gradients can be compared with those estimated by previous works, which found them to be in the range $[-0.3$, -0.2 ], with a typical uncertainty of 0.1 (see, e.g., IdM02). Both models $Z_{1}$ and $Z_{2}$, therefore, are in good agreement with previous $\nabla_{Z}$ estimates. The presence of overly shallow metallicity gradients in ETGs has often been invoked as a serious problem for monolithic scenarios of galaxy formation, since these models predict $\nabla_{Z}$ values in the range $[-1,-0.3]$ (Larson 1974; Carlberg 1984; Kawata 2001, hereafter KAW01). Models $Z_{1}$ and $Z_{2}$ show, however, that color gradients in galaxies in LRCs

TABLE 2

Parameters of Stellar Population Models for Color Gradients

\begin{tabular}{ccccccc}
\hline \hline Model & $Z_{i} / Z_{\odot}$ & $\begin{array}{c}T_{i} \\
(\mathrm{Gyr})\end{array}$ & $Z_{o} / Z_{\odot}$ & $\begin{array}{c}T_{o} \\
(\mathrm{Gyr})\end{array}$ & $\nabla_{Z}$ & $\nabla_{T}$ \\
\hline$Z_{1} \ldots \ldots \ldots$ & 1.0 & 11.4 & 0.6 & 11.4 & -0.22 & 0 \\
$Z_{2} \ldots \ldots \ldots$ & 1.0 & 11.4 & 0.4 & 11.4 & -0.4 & 0 \\
$T Z \ldots \ldots \ldots$ & 1.0 & 11.4 & 0.6 & 8.0 & -0.22 & -0.15 \\
$T \ldots \ldots \ldots$ & 1.0 & 11.4 & 1.0 & 8.0 & 0 & -0.15 \\
\hline
\end{tabular}




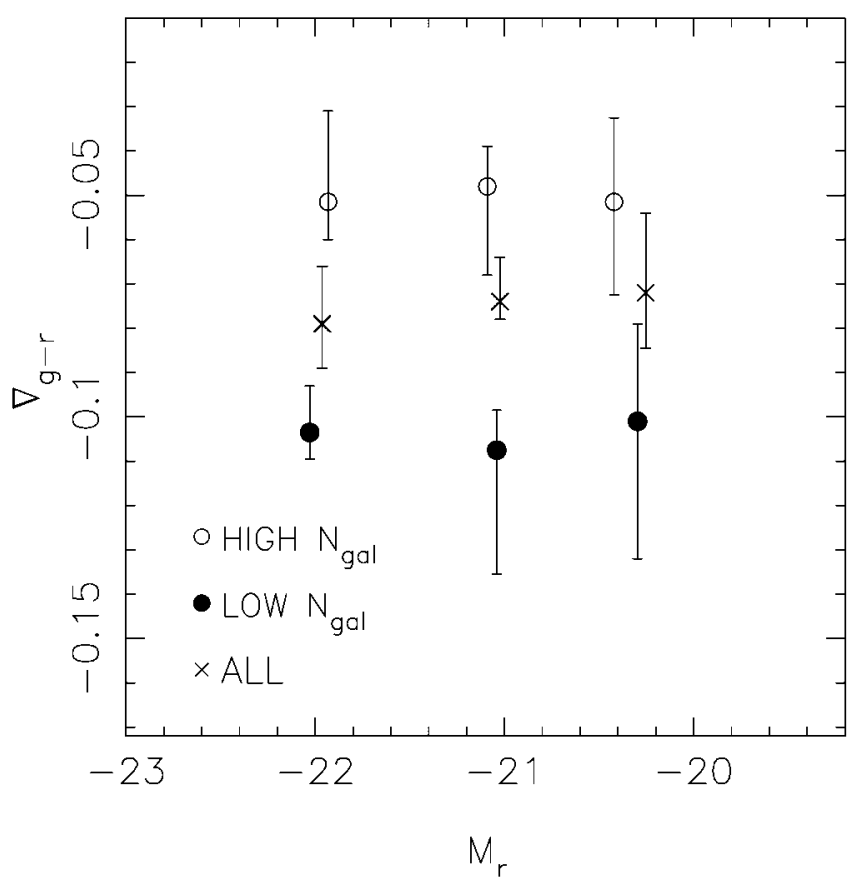

Fig. 3.-Mean values of $\nabla_{g-r}$ vs. galaxy absolute magnitude, $M_{r}$. Different symbols are used for HRCs, for LRCs, and for the whole sample, as shown in the lower left part of the figure. Error bars denote $1 \sigma$ uncertainties.

are fully consistent with expectations from the monolithic formation scenario, but the consistency is only marginal for HRCs. A natural candidate for the variation of the mean color gradient as a function of environment is galaxy merging, which is expected to flatten the stellar population gradient in galaxies (White 1980). Our results support a scenario in which ETGs have undergone increased (either major or minor) merger activity in richer rather than in poor clusters. Models $T$ and $T Z$, however, show that this interpretation is not unique. For example, the results for rich clusters can be equally well described by a metallicity gradient of -0.22 or by an age gradient of $-0.15($ model $T)$. The ratio of these gradients is $\nabla_{Z} / \nabla_{T} \sim 3 / 2$, which is simply the well-known age-metallicity degeneracy (Worthey et al. 1996). Model TZ shows that the richness dependence can also be explained by fixing the metallicity gra- dient of both HRCs and LRCs to that of model $Z_{1}$, provided that galaxies in LRCs have a younger stellar population in the outskirts, with an age gradient of about -0.15 . We note that a mild age gradient, of about -0.1 , would also be consistent with previous studies (Saglia et al. 2000; La Barbera et al. 2003). In a hierarchical merging picture, galaxies tend to develop a disk by accreting gas from the surrounding halo, with this mechanism being inhibited in high-density environments (Kauffmann et al. 1993). It would be conceivable, therefore, that a negative age gradient accounts for the steepening of the observed color gradients in LRCs.

A troublesome issue for galaxy formation theories is the absence of any sharp correlation between color gradient and galaxy luminosity. In a monolithic formation picture, the same galactic wind mechanism that is required to explain the colormagnitude relation of ETGs would produce a steepening of the metallicity gradient in more luminous galaxies (KAW01). Following KAW01 (model B in their Table 3), we expect a $\nabla_{g-r}$ change of about -0.08 in the luminosity range of Figure 3 , which is in clear disagreement with our data. We note that a steepening of the color gradients as a function of galaxy luminosity is expected if ETGs form through the dissipative merging of disk systems (Bekki \& Shioya 1999). The absence of correlation between gradient and luminosity could be explained by invoking (1) dissipationless merging, which could be more effective in flattening the metallicity gradient of massive galaxies, or (2) a steepening of age gradients in less massive galaxies, which could counteract the metallicity-luminosity relation. It is not obvious, however, which physical mechanism could drive this antagonistic behavior. The present data show that internal color gradients in ETGs probably result from a complex coordination of different physical processes. Crucial insights into these processes could be obtained by breaking the age-metallicity degeneracy, exploring color gradients for large samples of galaxies in the optical-NIR wave bands or at higher redshift.

R. de Carvalho, R. R. Gal, and S. G. Djorgovski acknowledge partial support from the Norris Foundation and the Ajax Foundation. We also thank the anonymous referee, whose comments have helped us to improve the paper. This work has been partially supported by the Italian Ministry of Education, University, and Research (MIUR) grant COFIN2004020323.

\section{REFERENCES}

Bekki, K., \& Shioya, Y. 1999, ApJ, 513, 108

Blanton, M. R., et al. 2003, ApJ, 594, 186

Bruzual, G., \& Charlot, S. 2003, MNRAS, 344, 1000

Carlberg, R. G. 1984, ApJ, 286, 403

De Propris, R., Colless, M., Driver, S. P., Pracy, M. B., \& Couch, W. J. 2004, MNRAS, 357, 590

Gal, R. R., de Carvalho, R. R., Brunner, R., Odewahn, S. C., \& Djorgovski, S. G. 2000, AJ, 120,540

Gal, R. R., de Carvalho, R. R., Lopes, P. A. A., Djorgovski, S. G., Brunner, R. J., Mahabal, A., \& Odewahn, S. C. 2003, AJ, 125, 2064

Idiart, T. P., Michard, R., \& de Freitas Pacheco, J. A. 2002, A\&A, 383, 30 (IdM02)

Kauffmann, G., White, S. D., \& Guiderdoni, B. 1993, MNRAS, 264, 201

Kawata, D. 2001, ApJ, 558, 598 (KAW01)

Kormendy, J., \& Djorgovski, S. G. 1989, ARA\&A, 27, 235

La Barbera, F., Busarello, G., Massarotti, M., Merluzzi, P., \& Mercurio, A. 2003, A\&A, 409, 21

La Barbera, F., Busarello, G., Merluzzi, P., Massarotti, M., \& Capaccioli, M. 2002, ApJ, 571, 790 (LBM02)
La Barbera, F., Merluzzi, P., Busarello, G., Massarotti, M., \& Mercurio, A. 2004, A\&A, 425, 797

Larson, R. B. 1974, MNRAS, 166, 585

Peletier, R. F., Davies, R. L., Illingworth, G. D., Davis, L. E., \& Cawson, M. 1990, AJ, 100, 1091 (PDI90)

Saglia, R. P., Maraston, C., Greggio, L., Bender, R., \& Ziegler, B. 2000, A\&A, 360,911

Tamura, N., Kobayashi, C., Arimoto, N., Kodama, T., \& Ohta, K. 2000, AJ, 119, 2134 (TKA00)

Tamura, N., \& Ohta, K. 2000, AJ, 120, 533

- 2003, AJ, 126, 596 (TaO03)

Visvanathan, N., \& Sandage, A. 1977, ApJ, 216, 214

White, S. D. 1980, MNRAS, 191, 1P

Worthey, G., Trager, S. C., \& Faber, S. M. 1996, in ASP Conf. Ser. 86, Fresh Views of Elliptical Galaxies, ed. A. Buzzoni, A. Renzini, \& A. Serrano (San Francisco: ASP), 203 